\title{
Gestão de qualidade de cursos a partir da percepção de estudantes egressos
}

\author{
Renato Cislaghi - UFSC - renato.cislaghi@ufsc.br \\ Silvia Modesto Nassar - UFSC - silvia.nassar@ufsc.br \\ Beatriz Wilges - UFSC - beatriz.wilges@gmail.com \\ Juliana Leonardi - UFSC - leonardiadm@gmail.com \\ Douglas Hiura Longo - UFSC - douglashiura@gmail.com
}

\begin{abstract}
Resumo: O objetivo desta pesquisa é implementar um modelo para gestão da qualidade de cursos com base em avaliações realizadas pelos estudantes egressos. Assim, indicadores-chave sobre a qualidade dos cursos são apresentados aos coordenadores como informações gerenciais e norteadoras. O modelo proposto é pautado por percepções de estudantes que já fizeram o curso e podem realizar comparações com as exigências do mercado de trabalho. Os egressos são considerados em quatro grupos: os que trabalham, os que trabalham e estudam, os que somente estudam e os que nem trabalham e nem estudam. Esses quatro grupos foram alinhados com à expectativa dos coordenadores em relação aos egresso obterem colocação no mercado de trabalho por um período variável de 6 a 24 meses. Ou seja, o módulo proposto atua a partir de parâmetros definidos por prévias manifestações dos coordenadores sobre suas expectativas quanto aos egressos serem absorvidos pelo mercado de trabalho. Assim, depois de um intervalo de seis meses, após a conclusão do curso, o módulo realiza o acompanhamento dos egressos durante um período de 2 anos. Os resultados desta pesquisa trazem informações significativas para apoiar a gestão quanto à melhoria do curso. Isto pode resultar em currículos de cursos mais focados e qualificados contemplando os atuais alunos do curso.
\end{abstract}

Palavras-chave: Acompanhamento de egresso. Apoio gerencial. Educação a distância.

\section{Management of the quality of courses from the perception of former students}

Abstract: The goal of this research is to develop a management model about the quality of courses based on evaluations made by former students. Thus, key indicators on the quality of courses are shown to the coordinators as management and guiding information. The proposed model is guided by the perception of students who have done the course and can make comparisons with the requirements of the labor market. The former students are distributed in four groups: those who work, those who work and study, those who just study and those who neither work nor study. These four groups were aligned with the expectations of the coordinators regarding the ability of the former students to get a placement in the labor market for a period ranging from 6 to 24 months. In other words, the proposed module operates from parameters set by previous manifestations of coordinators on their expectations of former students being absorbed by the labor market. So, after an interval of six months after completing the course, the module performs the monitoring of former students over a period of two years. The results of this research bring meaningful information to assist management in improving the course. This may result in more focused curriculum and in more qualified courses covering current students from the courses.

Keywords: Monitoring former students. Management support. Distance education. 


\section{Avaliação de cursos para gestão de qualidade}

A qualidade da educação se constitui em uma diretriz explicitada no Art. 2 §IV do Plano Nacional de Educação (PNE) 2014-2024 aprovado pela LEI N ${ }^{\mathrm{a}}$ 13.005, em 25 de junho de 2014. Um dos norteadores da qualidade de um curso é o Projeto Pedagógico de Curso (PPC) que expressa o desenvolvimento do curso de acordo com as diretrizes curriculares estabelecidas pelo MEC, considerando o perfil profissional dos concluintes. Desta forma, um estudante que obteve sua formação em um determinado curso de uma instituição de ensino pode contribuir com o curso, atualmente ofertado, e promover um processo de gestão comprometido com a qualidade e alinhamento com o mercado de trabalho.

Alguns autores mostram a necessidade do uso gerencial dos resultados avaliativos de forma a proporcionar uma reflexão do processo de ensino-aprendizagem com o objetivo de promover melhoria na qualidade dos cursos ofertados (CISLAGHI et al., 2012; FRIAS e TAKAHASHI, 2002; AMARAL et al., 2009 ; BRITO, 2008; MACHADO, 2010).

Cislaghi et al. (2012) esclarecem que, o processo de avaliação é fundamental e para que ele ocorra de forma satisfatória é indispensável a participação de todos os agentes envolvidos. Adicionalmente a utilização gerencial dos resultados por parte da coordenação complementa um conjunto de fatores críticos para o sucesso de um curso. Ainda neste contexto, é fundamental para a gestão do curso, observar os resultados e identificar possibilidades para garantir a qualidade do ensino.

Geralmente, a avaliação de cursos é direcionada aos alunos durante a frequência ao curso, isto é, durante o processo de ensino-aprendizagem (FRIAS e TAKAHASHI, 2002; AMARAL et al., 2009).

Por outro lado, alguns autores apontam para que a avaliação de cursos seja realizada após a saída do curso. Por exemplo, Brito(2008) e Machado(2010) destacam que a avaliação pelos concluintes ou egressos gera informações para revitalizar ou mesmo redirecionar a oferta de cursos pela instituição.

Brito (2008) destaca a importância que o egresso tem para a instituição e a necessidade de se fazer conter sua percepção no Projeto Pedagógico de Curso (PPC). E que, a definição do perfil do concluinte é de fundamental importância na compreensão do que é esperado, ao longo de trajetória do estudante pela instituição, alcançando as competências profissionais necessárias para o exercício da profissão.

O objetivo deste trabalho é implementar um modelo de gestão da qualidade de cursos com base em avaliações feitas pelos egressos. Assim, são visualizados indicadores através de informações pautadas na percepção de estudantes inseridos no mundo do trabalho.

Este artigo está organizado da seguinte forma: na seção dois são apresentados alguns trabalhos relacionados sobre avaliação de egressos. Na seção três é apresentado o módulo de gestão de qualidade de cursos pela percepção dos egresso, bem como seus procedimentos metodológicos desta pesquisa. A seção quatro apresenta os resultados da pesquisa. E por fim, são apresentadas as conclusões e referências.

\section{Trabalhos relacionados}

A proposta de investigar a percepção do egresso após sua formação é uma estratégia já identificada por diversas instituições que buscam um canal aberto de diálogo com seus estudantes. Assim, a instituição percebe que é capaz de oferecer cursos com qualidade 
porque possui informações relevantes sobre o mundo do trabalho a partir de seus egressos.

Entre algumas pesquisas que destacam-se o trabalho de Bardagi e Bizarro (2008), Meira e Kurcgant (2009), Lousada e Martins (2005), Machado (2010).

Nas pesquisas de Bardagi e Bizarro (2008), buscou-se conhecer a formação e as trajetórias de trabalho dos profissionais de psicologia formandos na UFRGS. Os resultados desta pesquisa convergiram ao encontro de novas diretrizes curriculares para o curso.

De acordo com Meira e Kurcgant (2009), os egressos passam por situações complexas que o levam a confrontar as competências desenvolvidas durante o curso com as requeridas no exercício profissional. Assim, eles são capazes de avaliar a estrutura pedagógica do curso e resgatar aspectos intervenientes desse processo.

Ainda segundo Lousada e Martins (2005), é de fundamental importância o planejamento e desenvolvimento de sistemas de acompanhamento de egressos como um mecanismo que permita às instituições a contínua melhoria de todo o planejamento e operação dessas organizações, particularmente do processo de ensino-aprendizagem.

Para Machado (2010), o processo de consulta aos egressos deve reunir características internas e externas de avaliação. As características internas referem-se a participação dos sujeitos que representam uma parte importante da comunidade acadêmica que, embora já tenham sido certificados, vivenciam de perto a realidade de seus cursos, com suas fragilidades e qualidades. Já nas características externas, a trajetória profissional vivida depois da formação, habilita esses ex-alunos a avaliarem a instituição com outro olhar, haja vista a experiência adquirida pelo exercício da profissão nos diferentes campos de trabalho.

Dessa forma, verifica-se a importância em coletar informações que expressem a percepção do egresso sobre o curso realizado (Módulo Egresso). Assim, após um período de conclusão do curso, é possível questionar sua opinião sobre a formação recebida, considerando sua atuação profissional. Essas informações podem servir como subsídios para tomadas de decisões contribuindo para a qualidade dos cursos e dos processos de ensino-aprendizagem.

\section{Módulos de gestão de qualidade de cursos por meio de egressos}

O módulo egresso faz um acompanhamento periódico dos concluintes, considerando aspectos-chave como: residência, empregabilidade, continuidade dos estudos e feedback para o curso:

O módulo egresso proposto foi construído para ser aplicado a partir de 6 meses de finalização do curso, em uma periodicidade semestral, ao longo de 24 meses, considerando, que o egresso possa estar em alguma das seguintes situações: a) trabalhando; b) estudando; c) nem trabalhando e nem estudando (desempregado); e d) trabalhando e estudando. Quando o egresso informa sua situação ele é direcionado a responder um questionário específico agrupado por quatro dimensões de análise.

Com o módulo proposto é possível adquirir dados e informações que favoreçam a qualidade de cursos a partir da atuação profissional dos egressos, possibilitando a verificação de estratégias adotadas pela gestão ou mesmo auxiliando na promoção de melhorias nos cursos técnicos. Desse modo, além de assegurar uma qualidade superior nos cursos em andamento, é possível obter informações sobre a trajetória do egresso com base nas avaliações realizadas por eles.

\subsection{Procedimento metodológico}


Esta seção descreve como a proposta do módulo foi especificada, considerando as fases de revisão da literatura, trabalhos relacionados, fonte de acesso aos dados, produção de instrumentos para investigação junto aos coordenadores gerais da instituição e coordenadores de curso na construção de meta para os cursos em relação a atuação dos egressos no mercado de trabalho. Além disso, também foram especificados os casos de uso do módulo proposto.

\subsubsection{Fonte de acesso aos dados}

Para construção e especificação do módulo egresso foram coletados dados sobre cursos técnicos do programa da Rede e-Tec (2015) no Sistema de Acompanhamento e Avaliação de Cursos (SAAS, 2015). O módulo egresso, descrito neste artigo, foi implementado como parte do SAAS.

A Secretaria de Educação a Distância/MEC, por meio da Rede e-Tec Brasil (2015) vem desenvolvendo ações visando o aprimoramento, ampliação e consolidação da oferta de ensino técnico na modalidade a distância, na periferia das áreas metropolitanas e no interior do país. Uma das principais ações desenvolvidas é o Sistema de Acompanhamento e Avaliação do e-Tec Brasil (SAAS, 2015), que possibilita a identificação das potencialidades e fragilidades da oferta de cursos e polos. A Tabela 1 sintetiza o total de dados cadastrados no sistema SAAS em agosto de 2014.

Tabela 1 - Dados cadastrados SAAS

\begin{tabular}{l|c}
\multicolumn{1}{c|}{ Cadastro } & n. \\
\hline Instituição & 58 \\
\hline Cursos & 297 \\
\hline Coordenador de Curso & 321 \\
\hline Coordenador Geral & 69 \\
\hline Coordenador Adjunto & 65 \\
\hline \multicolumn{2}{c}{ Fonte: SAAS (agosto de 2014) }
\end{tabular}

\subsubsection{Instrumento para definição de expectativa de metas}

Para estabelecer níveis de critérios sobre a inserção dos egressos no mundo do trabalho de forma a apoiar a gestão da qualidade dos cursos foi necessário extrair parâmetros a partir de estimativas de coordenadores. Assim, seis meses após a formação dos estudantes e durante um período de 2 anos de acompanhamento dos egressos, os coordenadores deveriam sinalizar suas expectativas quanto aos egressos serem absorvidos pelo mercado. Dessa forma, por meio de um questionário direcionado aos coordenadores gerais e de curso, solicitou-se que definissem o percentual que considerassem ideal para as seguintes situações: adequada, atenção e inadequada ao longo de 24 meses.

A pesquisa para caracterização dos indicadores de gestão foi composta por quatro questões: três objetivas que verificam junto aos coordenadores de curso e gerais a expectativa percentual, para acompanhamento do egresso para os períodos de: 6,12 , 18 e 24 . E uma questão qualitativa para comentários.

O questionário on-line foi elaborado e encaminhado por correio eletrônico para todos os coordenadores de curso técnicos e coordenadores gerais de todas as instituições cadastradas no sistema SAAS e pertencentes ao programa da Rede e-Tec. Assim, com base na Tabela 1 foram enviados 321 e-mails para coordenadores de curso e 69 e-mails para coordenadores gerais das instituições. O questionário ficou disponível durante sessenta dias. Assim, obteve-se um conjunto de 59 respostas, sendo 41 coordenadores de curso e 18 coordenadores gerais. Esta amostra atingiu o tamanho mínimo 
considerando uma população com 390 coordenadores, um nível de confiança de $90 \%$ e uma margem de erro de $10 \%$.

\subsubsection{Definição dos indicadores do módulo}

A partir dos parâmetros e metas definidos foi necessário elaborar a especificação dos casos de uso do sistema. Considerando as cores verde, amarelo e vermelho para sinalizarem a situação do egresso como adequada, que merece atenção e inadequada, respectivamente.

A situação "adequada" informa a presença de um cenário positivo ou satisfatório, e que as condições atuais podem ser mantidas. Essa situação é determinada exclusivamente quando o egresso está trabalhando ou quando ele, além de estar trabalhando, está estudando. Já uma situação intermediária, de "atenção", informa a presença tanto de aspectos positivos como negativos evidenciando que o curso precisa estar em "supervisão" para que não caia em cenários críticos. Essa situação é determinada quando o egresso está somente estudando. E na outra ponta, uma situação crítica caracterizada como "inadequada" informa que existem aspectos negativos, sendo necessárias ações imediatas para o bom desempenho do curso. Nessa situação, o egresso não está nem trabalhando e nem estudando.

Os resultados da pesquisa junto aos coordenadores foram relevantes porque são capazes de sintetizar em valores percentuais para cada situação (adequada, atenção e inadequada). Considerando ainda, um período de 24 meses de acompanhamento, iniciado 6 meses após a formação dos estudantes. Assim, esse período poderia ser suficiente para que o egresso estivesse vinculado a um emprego. Sendo assim, o Quadro 1 apresenta os indicadores com a média dos valores percentuais estimados de acordo com os coordenadores.

Quadro 1: Resultado em percentuais dos indicadores pelos coordenadores

\begin{tabular}{|c|c|c|c|c|c|c|c|c|c|c|c|c|}
\hline Situação & \multicolumn{4}{|c|}{ ADEQUADA } & \multicolumn{4}{|c|}{ ATENÇÃO } & \multicolumn{4}{|c|}{ INADEQUADA } \\
\hline \multirow{2}{*}{$\begin{array}{c}\text { Perguntas da } \\
\text { pesquisa }\end{array}$} & \multicolumn{4}{|c|}{$\begin{array}{l}\text { 1) Qual sua expectativa de } \\
\text { percentual mínimo de egressos } \\
\text { que consigam uma colocação no } \\
\text { mundo do trabalho, para cada } \\
\text { um dos seguintes períodos: }\end{array}$} & \multicolumn{4}{|c|}{$\begin{array}{l}\text { 2) Qual o percentual máximo } \\
\text { de egressos que se encontrem } \\
\text { somente Estudando, para cada } \\
\text { um dos seguintes períodos: }\end{array}$} & \multicolumn{4}{|c|}{$\begin{array}{l}\text { 3) Qual o percentual máximo } \\
\text { de egressos que se encontrem } \\
\text { desempregados, para cada um } \\
\text { dos seguintes períodos: }\end{array}$} \\
\hline & 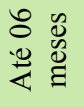 & 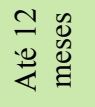 & 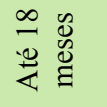 & 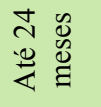 & \begin{tabular}{ll}
8 & 0 \\
0 & 0 \\
0 & 0 \\
\multirow{2}{*}{} & $\Xi$
\end{tabular} & 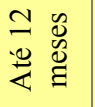 & 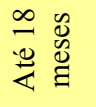 & 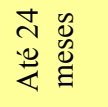 & \begin{tabular}{ll}
8 & 0 \\
0 & 0 \\
0 & 0 \\
\multirow{2}{*}{} & $\Xi$
\end{tabular} & 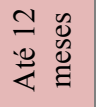 & 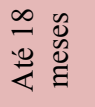 & 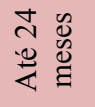 \\
\hline $\begin{array}{c}\text { Valores } \\
\text { Percentuais }\end{array}$ & $30 \%$ & $40 \%$ & $50 \%$ & $55 \%$ & $35 \%$ & $35 \%$ & $30 \%$ & $30 \%$ & $35 \%$ & $30 \%$ & $25 \%$ & $20 \%$ \\
\hline
\end{tabular}

Assim, em uma situação "adequada", os coordenadores consideraram que, em seis meses após o término do curso, pelo menos $30 \%$ dos egressos estejam trabalhando ou trabalhando e estudando; em doze meses, pelo menos $40 \%$ dos egressos estejam vinculados no mundo trabalho e assim sucessivamente. Já em uma situação de "atenção" os coordenadores consideraram um índice menor ou igual a 35\%, entre seis a doze meses para os egressos que se encontram somente estudando, após a conclusão do seu curso; e um índice menor ou igual a 30\% entre dezoito e vinte e quatro meses. E por fim, os coordenadores consideraram uma situação "inadequada" um índice maior ou 
igual a $35 \%$ nos primeiros seis meses, para egressos que não estejam trabalhando e nem estudando, após a conclusão do seu curso.

\section{Resultados gerais e discussões sobre os egressos}

Esta seção apresenta de forma resumida alguns resultados comparativos do módulo egresso. Os resultados apresentados ilustram avaliações aplicadas no período de 2014/2, caracterizando seis meses de conclusão do curso. Neste módulo, os egressos são considerados em quatro grupos: trabalhando; estudando; trabalhando e estudando; e nem trabalhando nem estudando.

Inicialmente o sistema questiona os egressos quanto sua ocupação atual. Os resultados dessa questão na coleta de 2014/2, para formandos de 2013/2, são apresentados na Figura 1.

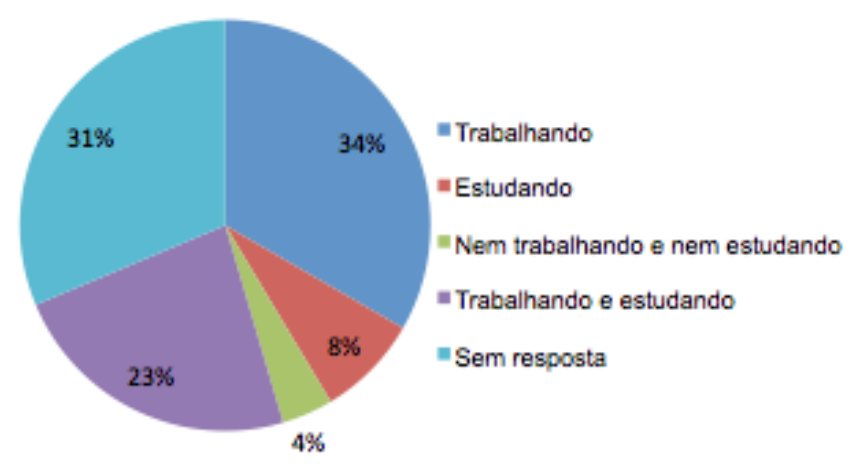

Figura 1 - Distribuição percentual da ocupação dos egressos após seis meses.

Dentre os cinco tópicos do módulo egresso cada grupo (trabalhando, trabalhando e estudando, estudando e nem trabalhando e nem estudando) respondeu um conjunto de questões específicas. A seguir ilustra-se o tópico "Feedback para o curso" por meio da questão sobre a avaliação da capacitação profissional recebida no curso.

\subsection{Resultados para o grupo trabalhando}

A avaliação da capacitação profissional recebida pelos egressos que estão trabalhando é apresentada na Figura 2.

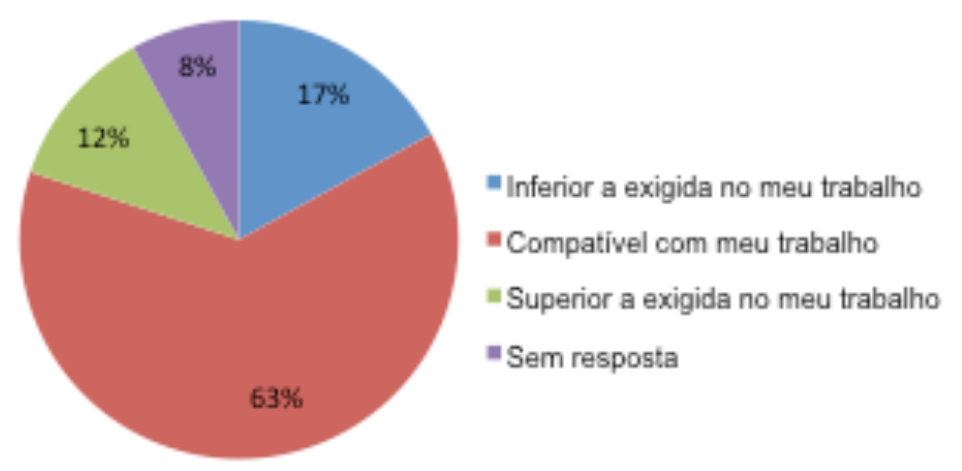

Figura 2 - Capacitação recebida pelos egressos que estão trabalhando

Dos 323 respondentes que estão trabalhando, a maioria (63\%) avaliou a capacitação profissional recebida como sendo compatível com seu trabalho. Apenas $12 \%$ afirmaram ser superior a exigida no seu trabalho. E $17 \%$ informaram que a capacitação recebida foi inferior a exigida no ambiente de trabalho.

\subsection{Resultados para o grupo trabalhando e estudando}

A mesma questão sobre a capacitação profissional recebida pelos egressos que estão trabalhando e estudando é apresentada na Figura 3. 


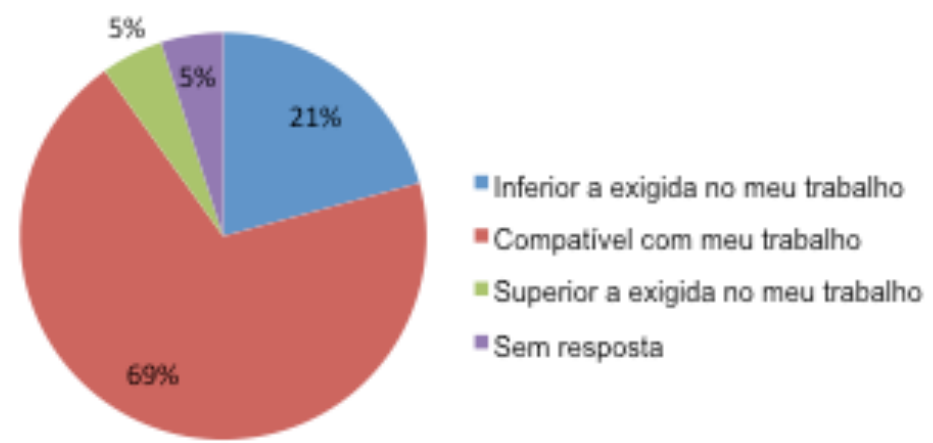

Figura 3 - Capacitação recebida pelos egressos que estão trabalhando e estudando

Entre 242 respondentes que estão trabalhando e estudando, a maioria (70\%) avaliaram a capacitação profissional recebida como sendo compatível com seu trabalho. Apenas 5\% afirmaram ser superior a exigida no seu trabalho. E $21 \%$ informaram que a capacitação recebida foi inferior a exigida no ambiente de trabalho.

\subsection{Resultados para o grupo estudando}

A mesma questão sobre a capacitação profissional recebida pelos egressos que estão somente estudando é apresentada na Figura 4.

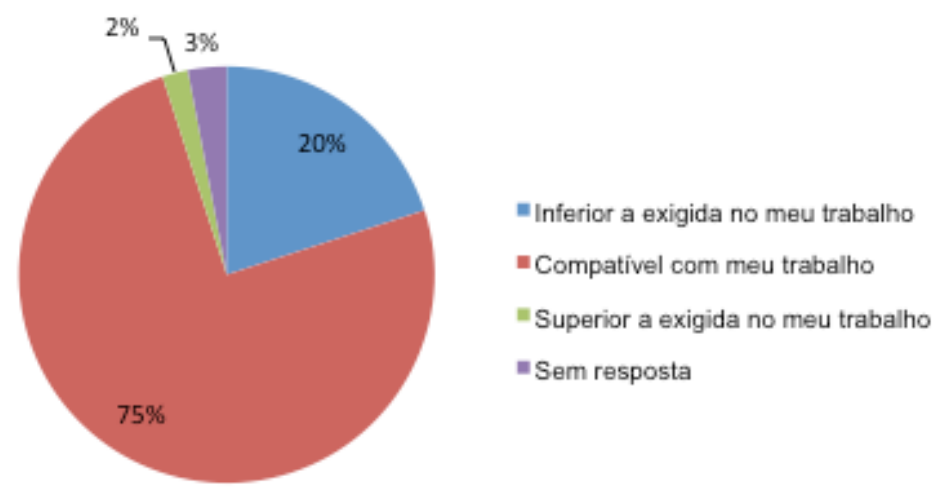

Figura 4 - Capacitação recebida pelos egressos que estão estudando

Dos 89 respondentes que estão estudando, a maioria (75\%) avaliou a capacitação profissional recebida como sendo compatível com o mundo do trabalho. Apenas 3\% afirmaram ser superior a exigida no mundo do trabalho. E $20 \%$ informaram que a capacitação recebida foi inferior a exigida no mundo do trabalho.

\subsection{Resultados para o grupo nem trabalhando e nem estudando}

A mesma questão sobre a capacitação profissional recebida pelos egressos que estão trabalhando e estudando é apresentada na Figura 5.

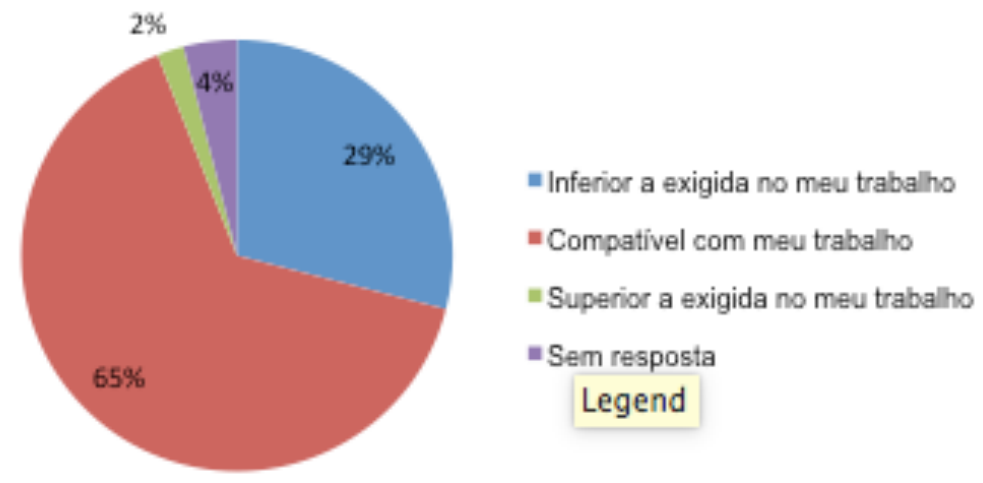

Figura 5 - Capacitação recebida pelos egressos que não estão trabalhando e nem estudando 
Dos 48 respondentes que estão trabalhando e estudando, a maioria (65\%) avaliou a capacitação profissional recebida como sendo compatível com o mundo do trabalho. Apenas 2\% afirmaram ser superior a exigida no seu trabalho. E 29\% informaram que a capacitação recebida foi inferior a exigida no ambiente de trabalho.

\subsection{Comentários dos egressos para melhorar o curso}

Nesta seção são apresentados comentários para melhorar os cursos a partir da percepção dos estudantes egressos dos diferentes grupos - trabalhando, estudando, trabalhando e estudando, e nem trabalhando nem estudando:

- "Mais aulas práticas para adquirir conhecimento na área do curso".

- "Em relação ao questionamento do feedback, a formação prática, apesar de estar adequada ao compromisso do curso, não que ela fosse insuficiente, mas deveria melhorar, como por exemplo: montar uma empresa virtual e, mesmo a distância elou presencial, colocar em prática os ensinamentos técnicos contábeis, administrativos, financeiros, etc. para fixar o aprendizado".

- "É necessário ajuda da instituição para arrumar um estágio para os alunos se colocarem no mercado".

- "Tutores mais qualificados e programas atuais, aulas laboratoriais com tutores presencias para prática do aprendizado".

- "Deve-se considerar o que o mercado de trabalho está exigindo de um profissional para adaptar o curso, inclusive no ensino prático".

- "Ressalto a importância das aulas práticas, para que haja uma aprendizagem significativa, pois é imprescindivel relacionar a teoria com a prática. Sugiro que as aulas práticas sejam valorizadas e que sejam mais praticadas. Comentei isso, pois quando cursei o Curso Técnico em Manutenção e Suporte em Informática. As aulas práticas eram pouco ofertadas".

\subsection{A interface de acompanhamento dos egressos}

A interface elaborada para a acompanhamento dos egressos em relação as turmas que finalizaram o curso é apresentada em um painel (Figura 6). Este painel pode ser visualizado por todos os coordenadores dos cursos respectivos.

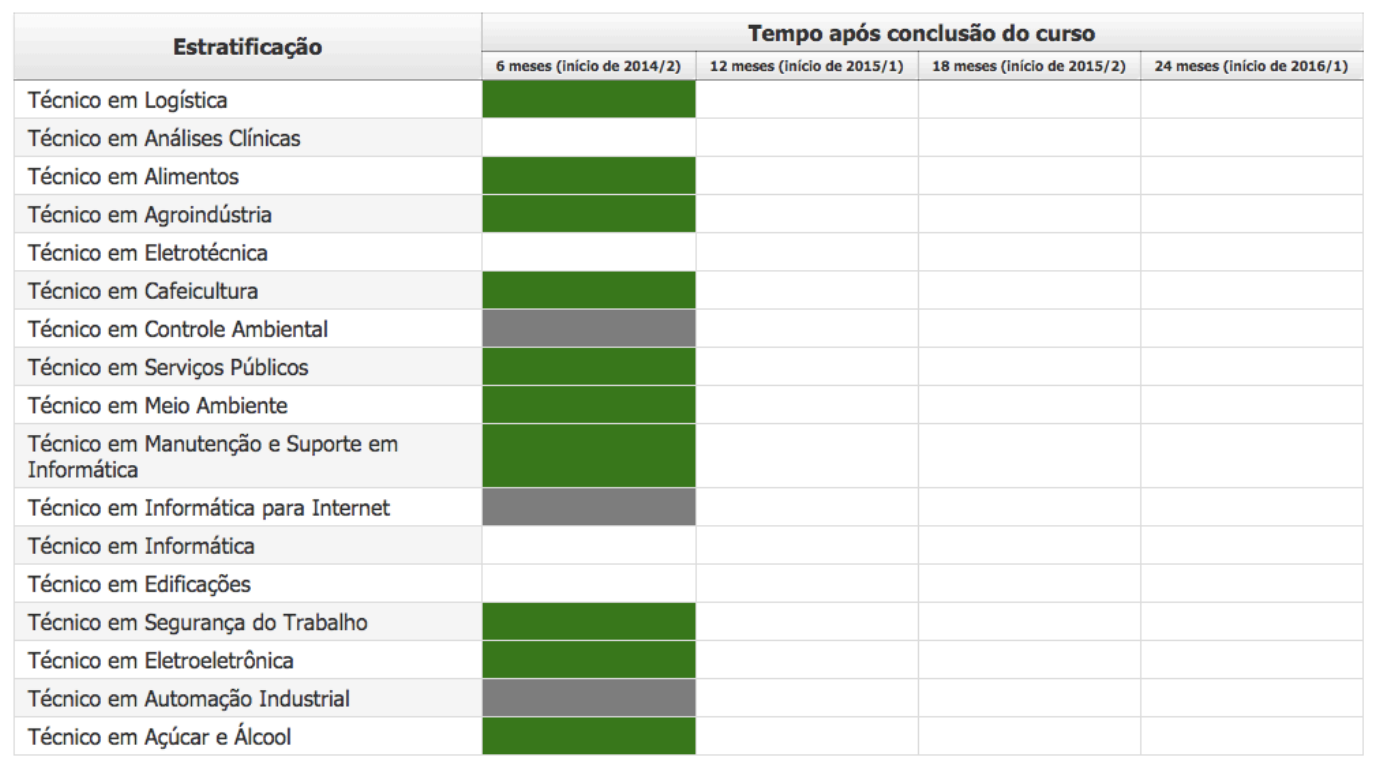

Figura 6 - Painel de acompanhamento por curso

Como este módulo possui uma coleta de dados com periodicidade semestral, e busca acompanhar a trajetória dos egressos durante vinte e quatro meses após a 
conclusão do curso, atualmente foi possível observar apenas os dados referentes aos primeiros seis meses de conclusão do curso. Essa interface do acompanhamento dos egressos permite analisar e acompanhar os egressos tanto por cursos como pelos eixos (Figura 7).

\begin{tabular}{|c|c|c|c|c|}
\hline \multirow{2}{*}{ Estratificação } & \multicolumn{4}{|c|}{ Tempo após conclusão do curso } \\
\hline & 6 meses (inicio de 2014/2) & 12 meses (inicio de 2015/1) & 18 meses (início de 2015/2) & 24 meses (iníio de 2016/1) \\
\hline \multicolumn{5}{|l|}{ Segurança } \\
\hline \multicolumn{5}{|c|}{ Controle e Processos Industriais } \\
\hline \multicolumn{5}{|l|}{ Produção Industrial } \\
\hline \multicolumn{5}{|l|}{ Ambiente e Saúde } \\
\hline \multicolumn{5}{|l|}{ Gestão e Negócios } \\
\hline \multicolumn{5}{|l|}{ Informação e Comunicação } \\
\hline \multicolumn{5}{|l|}{ Recursos Naturais } \\
\hline \multicolumn{5}{|l|}{ Produção Alimentícia } \\
\hline Infraestrutura & & & & \\
\hline
\end{tabular}

Figura 7 - Painel de acompanhamento por eixo

A legenda dos painéis das Figuras 6 e 7 são definidas pelas situações: adequada os egressos estão trabalhando ou trabalhando e estudando sinalizados em verde; atenção - os egressos estão somente estudando sinalizado em amarelo; inadequada - os egressos não estão nem trabalhando e nem estudando, sinalizado em cinza - duas situações muito próximas ou todas as situações mencionadas muito similares (portanto, inconclusivo); e branco - quando não existem informações, isto é, não houve respondentes.

\section{Considerações finais}

Quanto às expectativas de inserção no mundo do trabalho observou-se que $56 \%$ dos egressos estão trabalhando, $8 \%$ estão estudando, e $4 \%$ estão desempregados. Estes percentuais atendem aos indicadores apontados pelos coordenadores gerais e de cursos das instituições ofertantes.

$\mathrm{Na}$ avaliação sobre a capacitação profissional recebida ser compatível com o mundo do trabalho o percentual variou de $63 \%$ a $75 \%$ dos egressos. No entanto, observou-se um percentual que varia de $17 \%$ a $29 \%$ onde os egressos apontam para a necessidade de tornar o curso mais compatível com o mundo do trabalho.

Estes dois resultados, dentre os muitos que podem ser obtidos, no módulo egresso mostram que acompanhar o concluinte traz informações altamente significativas para apoiar a gestão quanto à melhoria do curso. Isto pode resultar em currículos de cursos mais focados e qualificados contemplando os atuais alunos do curso em avaliação.

Todos os resultados do módulo egresso podem ser obtidos por meio de consultas on-line tanto em máquinas desktops, quanto em dispositivos móveis como smartphones ou tablets. A atual versão do módulo foi planejada para gerar resultados de avaliações sobre a referência geográfica do polo. Assim, ícones no mapa do Brasil nas cores verde representam resultados satisfatórios no polo, amarelo resultados que merecem atenção, vermelho resultados que necessitam ser revistos urgentemente. Os resultados apresentados neste formato mais intuitivo permitem uma flexibilidade e uma leitura mais dinâmica. A flexibilidade se encontra na possibilidade de gerar consultas de interesse e realizar as análises até mesmo em modo off-line, e dinâmico porque é capaz de sintetizar qualquer resultado das avaliações no contexto georreferenciado.

Este módulo egresso é perfeitamente aplicável aos egressos de cursos presenciais. Finalmente, as instituições educacionais devem buscar a qualidade, a 
conscientização e o comprometimento no processo avaliativo de tal forma que os resultados possam ser utilizados para apoiar as ações para a melhoria contínua dos cursos e do processo de ensino-aprendizagem.

\section{Referências bibliográficas}

AMARAL, M. Antonio; ASSIS, KLeine K.; BARROS, Gilian C. Avaliação na EaD: contextualizando uma experiência do uso de instrumentos com vistas à aprendizagem. IX Congresso Nacional de Educação - EDUCERE III encontro Sul Brasileiro de Psicopedagogia. Out. 2009. Disponível em: http://www.pucpr.br/eventos/educere/educere2009/anais/pdf/3259_1706.pdf

BARDAGI, Marucia P.; BIZARRO, Lisiane. Avaliação da formação e trajetória profissional na perspectiva de egressos de um curso de Psicologia. Psicologia Ciência e Profissão, V. 28, N. 2, p.304-315, 2008. Disponível em http://www.scielo.br/pdf/pcp/v28n2/v28n2a07

BRITO, Marcia R. F de. O SINAES e o ENADE: da concepção à implantação. Avaliação, Campinas; Sorocaba, SP, v. 13, n. 3, p. 841-850, nov. 2008. Disponível em: http://www.scielo.br/pdf/aval/v13n3/14.pdf.

CISLAGHI, Renato; WILGES, Beatriz; NASSAR, Silvia Modesto; GONÇALVES, Wesley; CATAPAN, Araci Hack; OHIRA, Masanao. Sistema de acompanhamento e avaliação de cursos EaD: resultados avaliativos do AVEA, do curso, corpo discente e polo. IX Congresso Brasileiro de Ensino Superior a Distância - ESUD. Recife, 2012.

FRIAS, Marcos A.; TAKAHASHI, Regina T. Avaliação do processo ensinoaprendizagem: seu significado para o aluno de EM de enfermagem. Rev. Esc. Enferm. USP 2002; 36(2): 156-63. Disponível em: http://www.scielo.br/pdf/reeusp/v36n2/v36n2a07.pdf

LOUSADA, Ana Cristina Z.; MARTINS, Gilberto de A. Egressos como fonte de informação à gestão dos cursos de Ciências Contábeis. Revista Contabilidade \& Finanças, V.16, N.37, Jan./Apr. 2005. http://www.scielo.br/scielo.php?pid=S151970772005000100006\&script=sci_arttext

MACHADO, Geraldo Ribas. Perfil do egresso da Universidade Federal do Rio Grande do Sul. Tese de doutorado em Educação da Faculdade de Educação da Universidade Federal do Rio Grande do SUL - URGS. Porto Alegre, 2010. http://www.lume.ufrgs.br/bitstream/handle/10183/24186/000744974.pdf?sequence=1

MEIRA, Dyrce D. M.; KURCGANT, Paulina. Avaliação de curso de graduação segundo egressos. Revista Escola de Enfermagem da USP, V. 43, N. 2, p. 481-485, 2009. Disponível em http://www.scielo.br/pdf/reeusp/v43n2/a31v43n2.pdf

REDE e-Tec Brasil. Disponível em: http://redeetec.mec.gov.br/. Acesso: 10 jan. de 2015. 